\title{
The Role of nanoscience and technology in medicine and agriculture: awareness implication for health systems and food safety issues
}

\begin{abstract}
The problems of diseases resistance to drugs in Medicine and low productivity in Agriculture have led to alternatives for improvements in health care systems and food safety issues. Nanotechnology is currently the chosen option for preparation and delivery of human drugs, pesticides and herbicides among other applications. Agriculture and medicine are competitive in sustenance of life without one substituting the other. This review paper therefore, examined some of the positive and negative effects of nanotechnology application in life and the awareness implication. Nanoparticles (NPs) system could be used for several routes of administration including oral, nasal, parenteral, intra-ocular benefits over free antimicrobial agents. The use of nanomaterials in the production of goods and drug formulations have shown superior properties compared with their conventional counterparts, due to their distinctive nanoscale features. Nanoparticles (NPs) have also consistently been reported to decrease infection, reduce scar tissue growth, and promote bone growth among other benefits. Nanotechnology may have concrete solutions against many agriculture-related problems like insect pest management, adverse effects of chemical pesticides, development of improved crop varieties. As with any other technology, controversy surrounding nanotechnology is no exception in a heterogeneous society. The toxic effects of antimicrobial NPs on central nervous system (CNS) are still unknown, interactions of NPs with the cells and tissues in CNS were poorly understood. Again, NPs represent size-specific properties that limit the use of currently available in vitro experiments in a general way, and there was no standardized definition for NP dose in mass, number, surface area, and biological samples (e.g., blood, urine, and inside organs that guarantee safety limit). Educating and training of consumers through seminars and workshops is recommended. National governments especially in developing countries are advised to mount regulating agencies that will be responsible for awareness creation and achieving optimum utilization of new products.
\end{abstract}

Keywords: Nanoscience, Technology, Medicine, Agriculture, Health system, Food safety, Awareness
Volume 5 Issue 4 - 2017

\author{
Eze Simon C,' Eze DN, ${ }^{2}$ Onu FM ${ }^{3}$ \\ Department of Crop Science, University of Nigeria, Nigeria \\ 2Department of Human Kinetics and Health Education, \\ University of Nigeria, Nigeria \\ 'Department of Agricultural and Bio- resources Education, \\ University of Nigeria, Nigeria
}

Correspondence: Eze Simon C, Department of Crop Science, University of Nigeria, Nsukka, Enugu State, Nigeria Emailsimon@unn.edu.ns

Received: April 20, 2017 | Published: May 02, 2017

\section{Introduction}

Medicine and Agriculture appear to be the earliest knowledge based theory and practice which man developed with a view to sustain his life on earth. All medicines are of plant origin and early man probably selected some of the plants as medicine because of their efficacy in health care system. World Health Organization ${ }^{1}$ introduced the concept of essential medicines as those that satisfy the priority health care needs of the people. Experience has shown that careful selection of a limited range of essential medicines results in a higher quality of care, better management of medicines (including improved quality of prescribed medicines), and a more cost-effective use of available health resources. The World Medicines Situation Report 2004 of the World Health Organization (WHO) according to ${ }^{2}$ pointed out that approximately $67 \%$ of the population lives without an access to essential medicines. Agriculture stated according to history when seeds thrown away by man regenerated into a new plant and the man took good care and at the same time domesticated some of the animals he prayed on. By way of definition according to, ${ }^{3}$ agriculture involves design and management procedures that work with natural processes to conserve all resources, promote agro ecosystem resilience and self regulation, minimize waste and environmental damage, while maintaining or improving farm productivity and profitability. The development and growth of agriculture is highly dependent on its sustainability in line with ${ }^{4}$ assertion that sustainable agriculture systems are those that are economically viable and meet society's need for safe and nutritious food while maintaining or enhancing natural resources and the quality of the environment for future generations. The hallmark of these definitions is the harmony between agriculture and medicine in maintaining buoyancy and dynamism for meeting basic human needs and protection and conservation of natural resources.

Medicine and Agriculture are specialty areas that are most essential in human existence. The changes in ecosystem including increasing human population and decreasing natural resources give birth to search for alternative ways of improving the two subjects in order to meet the food need and health care systems of the teaming population. Again, the current development of diseases and pathogen resistance to pesticides and fungicides by pest and pathogens has called for search of improvement in drug delivery to the target organisms. ${ }^{5}$ Nanomaterials of inorganic and organic origins have been used for nanoparticles (NPs) synthesis by a variety of physical and chemical methods.

Synthesis of NPs as described by ${ }^{6}$ involves size reduction by topdown methods such as milling, high pressure homogenization and sonication, while bottom-up processes involve reactive precipitation and solvent displacement. Among inorganic materials, metal oxide NPs such as, $\mathrm{TiO} 2, \mathrm{ZnO}, \mathrm{AgO}$ and $\mathrm{MgO}$ are of particular interest 
as they are physically and optically stable with turnable optical properties. ${ }^{7}$ The application of NPs have been used in a number of ways to reduce disease levels and prevent the development and spread of pathogens, thus preserving yield and quality crop products. Abdelbasset et al. ${ }^{8}$ reported that both chitin and chitosan nanoparticles have demonstrated antiviral, antibacterial, and antifungal properties, and have been explored for many agricultural uses.

Studies by Bin $^{9}$ reported that the application of Nanotechnology in pesticide and drug delivery has the potential for efficient delivery of chemical and biological pesticides using nano-sized preparations or nano materials formulations. According to their report, the benefits of nano materials based formulations include but are not limited to the improvement of efficacy due to higher surface area, higher solubility, induction of systemic activity due to smaller particle size and higher mobility and lower toxicity due to elimination of organic solvents in comparison to conventionally used pesticides and their formulations. In another study by, ${ }^{10}$ both silver and organic nanoparticles (NPs) enhanced the efficacy and stability of whole cells, enzymes and other natural products used. Similarly, a controlled release of nanoclays engineered by surface coating with different polymers that manipulated electrostatic interactions between the chemical load and clay particles that resulted in efficient delivery has been reported. ${ }^{11}$ Sometimes when new technologies are developed, adoption becomes a problem and on the other hand, people may adopt a new technology without knowing the implication. The synthetic nano-based foods and medicines have in recent time taken prominent places in their utility in our society today. In some quarters, food is regarded as medicine in the sense that a hungry person is almost sick and cannot produce in his/her capacity while in some other area, food crops are medicinal plants and are used to treat sick people. Agriculture and medicine appear to be competitive in sustenance of life without substituting each other. This paper therefore, examines the role and significance of Nanoscience in the development and growth of these subjects and effects on health systems and food safety.

\section{Positive effects of nanoparticles in drug delivery}

Antibacterial properties of the metallic nanoparticles have been investigated and found useful in the control of Staphylococus aureus, had no toxic chemicals and no adverse effects on food. ${ }^{12}$ It has been applied for use in wound dressing, water purification, catheters and various house hold products due to their antimicrobial activities. ${ }^{13}$ The pathophysiological and anatomical changes of the affected tissues in a disease state offer many possibilities for the delivery of various nanotechnology-based products. ${ }^{14}$ Research studies by ${ }^{15}$ showed that bacteria gained antibiotic resistance due to three reasons namely:

1. Modification of active site of the target resulting in reduction in the efficiency of binding of the drug

2. Direct destruction or modification of the antibiotic by enzymes produced by the organism

\section{Efflux of antibiotic from the cell.}

Application of Nanoparticles (NPs) that targeted antimicrobial agents to the site of infection, so that higher doses of drug could be given at the infected site significantly reduced existing resistance mechanisms with fewer harmful effects upon the patient. ${ }^{16}$ It has been shown that nanoparticles when targeted actively contained ligands (e.g. antibodies) that bind receptors (e.g. antigens) at sites of infection. ${ }^{17}$

In a study of treating infectious disease with nanomaterials, ${ }^{18}$ noted that the use of NPs as delivery vehicles for antimicrobial agents suggested a new and promising model in the design of effective therapeutics against many pathogenic bacteria. According their finding, antimicrobial NPs proposed several clinical advantages:

1. The surface properties of nanoparticles could be changed for targeted drug delivery for e.g. small molecules, proteins, peptides, and nucleic acids loaded nanoparticles were efficiently targeted to special tissue types.

2. Nanocarriers could overcome solubility or stability issues of the drug and minimize drug-induced side effects.

3. Using nanotechnology could possibly achieve co-delivery of two or more drugs or therapeutic modality for combination therapy.

It has been reported that NP-based antimicrobial drug delivery was promising in overcoming resistance to common antibiotics developed by many pathogenic bacteria while administration of antimicrobial agents using NPs led to tremendous progress in therapeutic index thereby extending drug circulation (i.e., extended half-life), and achieved controlled drug release. ${ }^{19}$ The research report by ${ }^{20}$ indicated that NPs system could be used for several routes of administration including oral, nasal, parenteral, intra-ocular showing that antimicrobial NPs are of great interest as they provide a number of benefits over free antimicrobial agents. The use of nanomaterials in the production of goods and drug formulations according to ${ }^{21}$ had been shown to have superior properties compared with their conventional (or micron structured) counterparts, due to their distinctive nanoscale features and the new physical properties that affect their original characteristics. Again, nanomaterials have consistently been reported to decrease infection, reduce scar tissue growth, and promote bone growth. ${ }^{22}$ Fortunately, it has been observed through the use of both nanoparticles assembled as implants and as current implants that were modified to have nanostructured features. ${ }^{23,24}$ The new products have received increased attention, as there is no concern over nanoparticles becoming loose through mechanical wear and potential associated toxicity, which has yet to be largely determined -smooth implant.

\section{Negative effects of nanoparticles on drug delivery}

Although, numerous positive effects of nanoparticles in diverse formulations have been elucidated, negative effects should be the concern of our society due to probably some unforeseeable challenges in translating this exciting technology for clinical application. Profound knowledge according to ${ }^{25}$ about the potential toxicity of nanoantibiotics is needed to guarantee successful clinical translation. Earlier researchers, ${ }^{26}$ had noted that the toxic effects of antimicrobial NPs on central nervous system (CNS) are still unknown, and that the interactions of NPs with the cells and tissues in CNS were poorly understood. Again, ${ }^{27}$ stated that NPs represent size-specific properties that limit the use of currently available in vitro experiments in a general way, and that there was no standardized definition for NP dose in mass, number, surface area, and biological samples (e.g., blood, urine, and inside organs that guarantee safety limit. This means that the development of new characterization techniques that are not affected by NP properties as well as biological media is still in high demand. A research result carried out on drug delivery by ${ }^{28}$ showed that NPs usually have short circulation half-life due to natural defense mechanism of human body for eliminating them after opsonization by the mononuclear phagocytic system. Therefore, they suggested that the particles surfaces need to be changed to be hidden to opsonization.

\section{Nanoscience in agriculture}

The application of Nanoscience in agriculture, particularly crop agriculture is a welcome development due to high degrees of variable 
properties of most plants that aid the binding of nanomaterials in preparation of herbicides, pesticides, insecticides and fungicides used in the maintenance of plants health. It has been reported that most plants provide a non-toxic source of molecules with proven biological efficacy that are usually non-persistent in fresh water and soil. ${ }^{29}$ Incorporation of essential oils into a controlled-release nano formulation was capable of preventing rapid evaporation and degradation, enhanced stability and also maintained the minimum effective dosage/application. ${ }^{30}$ The researchers noted that essential oil from garlic loaded on polymer NPs $(240 \mathrm{~nm})$ coated with polyethylene glycol (PEG) showed effective insecticidal activity against adult Tribolium castaneum. ${ }^{31}$ The Sichuan Academy of Agricultural Science succeeded in developing nanopesticides $(<100 \mathrm{~nm})$ from a plant source, that had advantages of environmental protection, high efficiency and low toxicity, which had a direct effect on pest control practices. ${ }^{32} \mathrm{~A}$ research conducted in India, showed that silver nano particles (Ag NPs) synthesized using aqueous leaves extracts of Euphorbia prostrata were successfully used to control Sitophilus oryzae in rice and showed potential of being used as an ideal ecofriendly approach. ${ }^{33}$ The advantage of using plants for the synthesis of nano pesticides is that they are easily available, safe to handle and possess a broad variability of metabolites that may aid in reduction to nanoparticle size. As earlier stated that all medicines are of plant origin, a number of plants/crops are being currently investigated for their role in the nanoparticle usage and preparation was from Gold Nanoparticles with a size range of 2- 20 synthesized using live alfalfa plants was achieved. ${ }^{34}$

\section{Detection of plant pathogens using nanosensors}

Nanoscience has successfully made treatment of certain plant diseases caused by pathogenic organisms simple cost effective. Technique of detecting single bacterial cell was possible as reported by $^{35}$ in their study titled "Rapid bioassay for single bacterial cell quantitation using bio-conjugated nanoparticles" showed that pesticides such as atrazine, molinate, and chlorpyrifos are susceptible to degradation with nano sized zero valent iron (ZVI, 1-100 nm). Other approaches such as photocatalytic decomposition of pesticide residues using titanium doped with $\mathrm{Fe} 2 \mathrm{O} 3$ or other metals sprayed directly on crops or even incorporated into the pesticide formulation are promising. ${ }^{36}$ Layer-by-layer surface (LbL) nano-engineering is a novel strategy for direct surface modification of colloidal entities, which utilizes sequential adsorption of oppositely charged polyelectrolytes to form a complex assembly via electrostatic interactions.

\section{Detection of pesticides using nanobiosensors}

The development of diagnostic devices using nanoparticles for on-site monitoring allowed farmers to closely monitor environmental conditions for plant growth and protection. These detection systems can contribute to increased productivity and decrease the use of synthetic conventional agrochemicals (example, antibiotics, pesticides, nutrients) by early intervention. Application of nanomaterials was reported to have advantages such as measurement of more variables for greater sensitivity with less sample material required and faster detection rates, read-outs in real time and application of novel detection methodologies, for example electronic, colorimetric, fluorometric, and mass changes. ${ }^{37}$

Currently uni-molecular and array type of nano material based biosensors are being developed for detection of pesticides residues on consumables thereby indirectly improving the health system of man. ${ }^{38}$ Interaction of the target with the biosensor can be measured either directly or indirectly by recording the changes in color, fluorescence or electrical potential. In array technologies, multiple biomolecules are fixed to a substrate allowing multiple analytes to be measured simultaneously. The gold NPs based dipstick technique was developed and found suitable for the detection of several toxins in food and environments and can also be applied for rapid on-site testing of pesticides. ${ }^{38}$

\section{Field application of nano formulations}

In the field, the integration of biomolecules such as enzymes, metabolites or whole cells with nanostructures assisted plant breeders to develop hybrid systems that have numerous applications in many fields including agriculture. ${ }^{39}$ According to Matthews ${ }^{40}$ the mode of pesticide and fertilizer application influenced their efficiency and their impact on the environment. Constraints due to droplets were circumvented by using NPs encapsulated or nano-sized pesticides that contributed to efficient spraying and reduction of spray drift and splash losses.

According to Vijayakumar, ${ }^{41}$ the DNA coated NPs gained easy access to the plant cell due to its size that increased the transformation efficiency in both monocotyledonous Oryza sativa (rice), and dicotyledenous Nicotiana tabacum (tobacco) and Leucaena leucocephala (white lead tree). Moreover, plant-cell damage with the NPs was minimal with increased plant regeneration. Furthermore, it has earlier been demonstrated that a honeycomb surface functionalized mesoporous silica NP system with $3 \mathrm{~nm}$ pores capped with disulphide bond held gold NPs (10-15 nm) transported DNA and chemicals into isolated plant cells and intact leaves with the help of gene gun. ${ }^{42}$

\section{Conclusion}

Medicine and agriculture are such professional areas where man is constantly looking for ways of developing in tandem with human population growth, climate change and pest and pathogen resistance to existing drugs. Application of nanoscience appears to be the best option due to its numerous advantages such as:

1. Easy manipulation for different functions due its large surface area.

\section{Nanoparticles have no drug side effects and are non toxic.}

3. Using nanotechnology could possibly achieve co-delivery of two or more drugs or therapeutic modality for combination therapy.

4. Nanoparticles prepared with plant materials are always available and cost effective among other benefits.

However, Nanotechnology has the potential to revolutionize the existing technologies used in various sectors including medicine and agriculture in the near future. Its products can be used in a number of ways to reduce disease levels and prevent the development and spread of pathogen, thus promoting plants and animal health systems. As with any other technology, controversy surrounding nanotechnology is no exception in a heterogeneous society. Several concerns need to be addressed on different issues like food safety and beneficiaries of the technology. Therefore, it is high time we strengthened research in this direction to gather long term benefits from nanotechnology. However, governments and agencies should make a complex decision about the suitability of existing regulatory systems and determine whether new measures are needed for nanoparticles to be adopted into the market. Increasing agricultural productivity is necessary, while safety of the food in our health system is a major concern. Educating and training of consumers through seminars and workshops is recommended. 
National governments especially in developing countries are advised to mount regulating agencies that will be responsible for awareness creation and achieving optimum utilization of new products.

\section{Acknowledgments}

None.

\section{Conflicts of interest}

None.

\section{References}

1. Miedema K. Standardization of HbA1c and Optimal Range of Monitoring. Scand J Clin Lab Invest. 2005;240:61-72.

2. Peterson KP, Pavlovich JG, Goldstein D, et al. What is hemoglobin A1c? An analysis of glycated hemoglobins by electrospray ionization mass spectrometry. Clin Chem. 1998;44(9):1951-1958.

3. Kobold U, Jeppsson JO, Dulffer T, et al. Candidate reference methods for hemoglobin Alc based on peptide mapping. Clin Chem. 1997;43:1944-1951.

4. Koval D, Kašička V, Cottet H. Analysis of glycated hemoglobin A1c by capillary electrophoresis and capillary isoelectric focusing. Anal Biochem. 2011;413(1):8-15.

5. Reynolds TM, Smellie WS, Twomey PJ. Glycated haemoglobin (HbA1c) monitoring. BMJ. 2006;333(7568):586-588.

6. Xanthis A, Hatzitolios A, Koliakos G, et al. Advanced glycosylation end products and nutrition - a possible relation with diabetic atherosclerosis and how to prevent it. J Food Sci. 2007;72(8):R125-R129.

7. Koenig RJ, Peterson CM, Jones RL, et al. Correlation of glucose regulation and hemoglobin AIc in diabetes mellitus. $N$ Engl $\mathrm{J} \mathrm{Med.}$ 1976;295(8):417-420

8. Grundy SM, Benjamin IJ, Burke GL, et al. Diabetes and cardiovascular disease: a statement for healthcare professionals from the American Heart. Association Circulation. 1999;100(10):1134-1146.

9. Little RR. Glycated hemoglobin standardization-National Glycohemoglobin Standardization Program (NGSP) perspective. Clin Chem Lab Med. 2003;41(9):1191-1198.

10. Cagliero E, Levina EV, Nathan DM. Immediate feedback of HbAlc levels improves glycemic control in type 1 and insulin-treated type 2 diabetic patients. Diabetes Care. 1999;22(11):1785-1789.

11. Miller CD, Barnes CS, Phillips LS, et al. Rapid A1c availability improves clinical decision-making in an urban primary care clinic. Diabetes Care. 2003;26(4):1158-1163.

12. Wu X, Monnier VM. Enzymatic deglycation of proteins. Arch Biochem Biophys. 2003;419(1):16-24.

13. Szwergold BS, Howell S, Beisswenger PJ. Human fructosamine-3kinase: purification, sequencing, substrate specificity, and evidence of activity in vivo. Diabetes. 2001;50(9):2139-2147.

14. Depierre G, Van SE. Fructosamine 3-kinase, an enzyme involved in protein deglycation. Biochemical Society transactions. 2003;31(6):1354-1357.

15. Szwergold BS, Howell SK, Beisswenger PJ. Transglycation-a potential new mechanism for deglycation of Schiff's bases. Ann NY Acad Sci. 2005;1043:845-864.

16. Luthra M, Balasubramanian D. Nonenzymatic glycation alters protein structure and stability. A study of two eye lens crystallins J Biol Chem. 1993;268(24):18119-18127.
17. Jandeleit Dahm K, Cooper ME. The role of AGEs in cardiovascular disease. Curr Pharm Des. 2008;14(10):979-986.

18. Yan SF, D'Agati V, Schmidt AM, et al. Receptor for Advanced Glycation Endproducts (RAGE): a formidable force in the pathogenesis of the cardiovascular complications of diabetes \& aging. Curr Mol Med. 2007;7(8):699-710.

19. Peacock I. Glycosylated haemoglobin: measurement and clinical use. $J$ Clin Pathol. 1984;37(8):841-851.

20. Little RR, Wiedmeyer HM, JD England, et al. Interlaboratory standardization of measurements of glycohemoglobins. Clinical Chemistry. 1992;38(12):2472-2478.

21. Uwe Kobold, Jan Olof Jeppsson, Thomas Dülffer, et al. Candidate reference methods for hemoglobin A1c based on peptide mapping. Clinical Chemistry. 1997;43(10):1944-1951.

22. Mosca A, Goodall I, Hoshino T, et al. Global standardization of glycated hemoglobin measurement: the position of the IFCC Working Group. Clin Chem Lab Med. 2007;45(8):1077-1080.

23. Hanas R. Psychological impact of changing the scale of reported $\mathrm{HbA}(1 \mathrm{c})$ results affects metabolic control. Diabetes Care. 2002;25(11):2110-2111.

24. Hoelzel W, Weykamp C, Jeppsson JO, et al. IFCC reference system for measurement of hemoglobin A1c in human blood and the national standardization schemes in the United States, Japan, and Sweden: a method-comparison study. Clin Chem. 2004;50(1):166-174.

25. Cas Weykamp. HbA1c: a review of analytical and clinical aspects. Ann Lab Med. 2013;33(6):393-400.

26. Nathan DM, Turgeon H, Regan S. Relationship between glycated haemoglobin levels and mean glucose levels over time. Diabetologia. 2007;50(11):2239-2244.

27. Rohlfing CL, Wiedmeyer HM, Little RR, et al. Defining the relationship between plasma glucose and $\mathrm{HbA}(1 \mathrm{c})$ : analysis of glucose profiles and $\mathrm{HbA}(1 \mathrm{c})$ in the Diabetes Control and Complications Trial. Diabetes Care. 2002;25(2):275-278.

28. Nathan DM, Kuenen J, Borg R, et al. Translating the A1C assay into estimated average glucose values. Diab Care. 2008;31(8):1473-1478.

29. Padma Rajan, Mahipal Nera, Aravind Kumar Pavalura, et al. Comparison of glycosylated hemoglobin (HbA1C) levels in patients with chronic periodontitis and healthy controls. Dent Res $J$ (Isfahan). 2013;10(3):389-393.

30. Rodbard HW, Blonde L, Braithwaite SS, et al. American Association of Clinical Endocrinologists medical guidelines for clinical practice for the management of diabetes mellitus. Endocr Pract. 2007;13(1):1-68.

31. Huang ES1, Zhang Q, Gandra N, et al. The effect of comorbid illness and functional status on the expected benefits of intensive glucose control in older patients with type 2 diabetes: a decision analysis. $s$. 2008;149(1):11-19.

32. Saudek CD, Kalyani RR. Does comorbidity affect the benefit of intensive glycemic control in older patients with type 2 diabetes mellitus? Nat Clin Pract Endocrinol Metab. 2009;5(2):78-79.

33. Melissa Conrad Stöppler. HbA1c Test (Hemoglobin A1c). 2016.

34. Diazyme laboratories. Innovations in clinical Diagnostics. Direct Enzymatic HbA1c Methods. 2017.

35. Pohanka, Miroslav. Monoclonal and polyclonal antibodies production - preparation of potent biorecognition element. Journal of Applied Biomedicine (De Gruyter Open). 2009;7(3):115-121.

36. Hamwi A, Schweiger CR, Veitl M, et al. Quantitative measurement of $\mathrm{HbA} 1 \mathrm{c}$ by an immunoturbidimetric assay compared to a standard HPLC method. Am J Clin Pathol. 1995;104(1):89-95. 
37. Camargo JL, Gross JL. Conditions associated with very low values of glycohaemoglobin measured by an HPLC method. J Clin Pathol. 2004;57(9):346-349.

38. Maurice Owen. The glucose vs. HbA1c controversy. Canterbury Scientific, New Zealand. 2015.

39. Jaisson S, Desmons A, Renard B, et al. Analytical performances of a new enzymatic assay for hemoglobin A1c. Clin Chim Acta. 2014;434(1):48-52.
40. Liu L, Hood S, Wang Y, et al. Direct enzymatic assay for $\% \mathrm{HbA} 1 \mathrm{c}$ in human whole blood samples. Clin Biochem. 2008;41(7-8):576-583.

41. Stephen DS, Edwin GM. Reagent and test kit for determining glycosylated hemoglobin. 1981.

42. Douglas RB, William EB, Theresa LL, et al. Determination of glycated hemoglobin by fluorescence quenching. 1993. 